\title{
Set-valued mappings in partially ordered fuzzy metric spaces
}

\author{
Zahra Sadeghi', S Mansour Vaezpour ${ }^{2 *}$, Choonkil Park ${ }^{3 *}$, Reza Saadati ${ }^{4}$ and Calogero Vetro ${ }^{5}$
}

\author{
"Correspondence: vaez@aut.ac.ir; \\ baak@hanyang.ac.kr \\ 2Department of Mathematics, \\ Amirkabir University of Technology, \\ Tehran, Iran \\ ${ }^{3}$ Department of Mathematics, \\ Research Institute for Natural \\ Sciences, Hanyang University, Seoul, \\ 133-791, Korea \\ Full list of author information is \\ available at the end of the article
}

\begin{abstract}
In this paper, we provide coincidence point and fixed point theorems satisfying an implicit relation, which extends and generalizes the result of Gregori and Sapena, for set-valued mappings in complete partially ordered fuzzy metric spaces. Also we prove a fixed point theorem for set-valued mappings on complete partially ordered fuzzy metric spaces which generalizes results of Mihet and Tirado.
\end{abstract}

MSC: 54E40; 54E35; 54H25

Keywords: fixed point; coincidence point; set-valued mapping; partially ordered set; fuzzy metric space

\section{Preliminaries}

The concept of fuzzy metric space was introduced by Kramosil and Michalek [1] and the modified concept by George and Veeramani [2] (for other modifications see [3, 4]). Furthermore, the fixed point theory in this kind of spaces has been intensively studied (see [5-14]).

The applications of fixed point theorems are remarkable in different disciplines of mathematics, engineering, and economics in dealing with problems in approximation theory, game theory, and many others (see [15] and references therein).

In 2004 Rodríguez-López and Romaguera [16] introduced the Hausdorff fuzzy metric of a given fuzzy metric space in the sense of George and Veeramani on the set of non-empty compact subsets.

Some fixed point results for set-valued mappings on fuzzy metric space can be found in $[17,18]$ and references therein.

The aim of this paper is to prove a coincidence point and fixed point theorem on a partially ordered fuzzy metric space satisfying an implicit relation and another fixed point theorem. Our result substantially generalizes and extends the result of Gregori and Sapena [8] and results of Miheț [19] and Tirado [20] and also the result of Latif and Beg [21] for set-valued mappings in complete partially ordered fuzzy metric spaces. Implicit relations have been considered by several authors in connection with solving nonlinear functional equations (see [22-25]).

For the sake of completeness, we briefly recall some basic concepts used in the following.

Definition 1.1 [26] A binary operation $*:[0,1] \times[0,1] \rightarrow[0,1]$ is called a continuous $t$-norm if it satisfies the following conditions:

(1) * is associative and commutative, 
(2) $*$ is continuous,

(3) $a * 1=a$ for all $a \in[0,1]$,

(4) $a * b \leq c * d$ whenever $a \leq c$ and $b \leq d$, for each $a, b, c, d \in[0,1]$.

The three basic continuous $t$-norms are: (i) The minimum $t$-norm is defined by $a * b=$ $\min \{a, b\}$. (ii) The product $t$-norm is defined by $a * b=a b$. (iii) The tukasiewicz $t$-norm is defined by $a * b=\max \{a+b-1,0\}$.

Definition $1.2[27,28]$ (i) A $t$-norm $*$ is said to be Hadžić-type $t$-norm, if the family $\left\{*^{n}\right\}_{n \geq 0}$ of its iterates defined for each $s \in[0,1]$ by $*^{0}(s)=1, *^{n}(s)=\left(*^{n-1}(s)\right) * s$, for all $n \geq 0$, are equi-continuous at $s=1$, that is, given $\lambda>0$, there exists $\eta(\lambda) \in(0,1)$ such that for all $n \geq 0$

$$
1 \geq s>\eta(\lambda) \quad \Rightarrow \quad *^{n}(s)>1-\lambda
$$

The $t$-norm $*$, defined by $a * b=\min \{a, b\}$ is a trivial example of the $t$-norm of Hadžićtype, but there are other $t$-norms of Hadžić-type (see [27]).

(ii) If $*$ be a $t$-norm and $\left\{x_{n}\right\}_{n \geq 1}$ is a sequence of numbers in $[0,1]$, one defines recurrently $*_{i=1}^{n} x_{i}$ by $*_{i=1}^{1} x_{i}=x_{1}$ and $*_{i=1}^{n} x_{i}=*\left(*_{i=1}^{n-1} x_{i}, x_{n}\right), \forall n \geq 2 . *_{i=1}^{\infty} x_{i}$ is defined as $\lim _{n \rightarrow \infty} *_{i=1}^{n} x_{i}$ and $*_{i=n}^{\infty} x_{i}$ as $*_{i=1}^{\infty} x_{n+i}$.

If $q \in(0,1)$ is given, we say that the $t$-norm is geometrically convergent ( $g$-convergent) if $\lim _{n \rightarrow \infty} *_{i=n}^{\infty}\left(1-q^{i}\right)=1$.

The Lukasiewicz $t$-norm and $t$-norms of Hadžić-type are examples of $g$-convergent $t$ norms. Other examples be found in [28]. Also note that if the $t$-norm $*$ is $g$-convergent, then $\sup _{t<1} t * t=1$.

\section{Proposition 1.3 [28]}

(i) For $a * b \geq \max \{a+b-1,0\}$ the following implication holds:

$$
\lim _{n \rightarrow \infty} \underset{i=1}{*} x_{n+i}=1 \quad \Leftrightarrow \quad \sum_{n=1}^{\infty}\left(1-x_{n}\right)<\infty
$$

(ii) If $*$ is of Hadžić-type, then $\lim _{n \rightarrow \infty} *_{i=1}^{\infty} x_{n+i}=1$, for every sequence $\left\{x_{n}\right\}_{n \in \mathbb{N}}$ in $[0,1]$ such that $\lim _{n \rightarrow \infty} x_{n}=1$.

Definition 1.4 [2] A 3-tuple $(X, M, *)$ is called a fuzzy metric space if $X$ is an arbitrary non-empty set, $*$ is a continuous $t$-norm and $M$ is a fuzzy set on $X^{2} \times(0, \infty)$, satisfying the following conditions for each $x, y, z \in X$ and $t, s>0$ :

(FM-1) $M(x, y, t)>0$,

(FM-2) $M(x, y, t)=1$ for all $t>0$ if and only if $x=y$,

(FM-3) $M(x, y, t)=M(y, x, t)$,

(FM-4) $M(x, z, t+s) \geq M(x, y, t) * M(y, z, s)$

$(\mathrm{FM}-5) M(x, y, \cdot):(0, \infty) \rightarrow[0,1]$ is continuous.

Definition 1.5 [2] Let $(X, M, *)$ be a fuzzy metric space. A sequence $\left\{x_{n}\right\}$ in $X$ is called a Cauchy sequence, if, for each $\epsilon \in(0,1)$ and $t>0$, there exists $n_{0} \in \mathbb{N}$ such that $M\left(x_{n}, x_{m}, t\right)>$ 
$1-\epsilon$ for all $n, m \geq n_{0}$. A sequence $\left\{x_{n}\right\}$ in a fuzzy metric space $(X, M, *)$ is said to be convergent to $x \in X$ if $\lim _{n \rightarrow \infty} M\left(x_{n}, x, t\right)=1$ for all $t>0$. A 3-tuple $(X, M, *)$ is complete if every Cauchy sequence is convergent in $X$.

Lemma 1.6 [7] Let $(X, M, *)$ be a fuzzy metric space. Then $M(x, y, t)$ is non-decreasing with respect to $t$ for all $x, y \in X$.

Definition 1.7 [16] Let $(X, M, *)$ be a fuzzy metric space. $M$ is said to be continuous on $X^{2} \times(0, \infty)$, if

$$
\lim _{n \rightarrow \infty} M\left(x_{n}, y_{n}, t_{n}\right)=M(x, y, t)
$$

whenever a sequence $\left\{\left(x_{n}, y_{n}, t_{n}\right)\right\}$ in $X^{2} \times(0, \infty)$ converges to a point $(x, y, t) \in X^{2} \times(0, \infty)$, that is,

$$
\lim _{n \rightarrow \infty} x_{n}=x, \quad \lim _{n \rightarrow \infty} y_{n}=y, \quad \text { and } \quad \lim _{n \rightarrow \infty} M\left(x, y, t_{n}\right)=M(x, y, t) .
$$

Lemma 1.8 [16] Let $(X, M, *)$ be a fuzzy metric space. Then $M$ is continuous function on $X^{2} \times(0, \infty)$.

Definition 1.9 [6] Let $(X, M, *)$ be a fuzzy metric space. The fuzzy metric $M$ is triangular if it satisfies the condition

$$
\left(\frac{1}{M(x, y, t)}-1\right) \leq\left(\frac{1}{M(x, z, t)}-1\right)+\left(\frac{1}{M(z, y, t)}-1\right)
$$

for every $x, y, z \in X$ and every $t>0$.

Example 1.10 [2] Let $(X, d)$ be a metric space. Define $a * b=a b$ (or $a * b=\min \{a, b\})$ and for all $x, y \in X$ and $t>0$,

$$
M_{d}(x, y, t)=\frac{t}{t+d(x, y)} .
$$

Then $\left(X, M_{d}, *\right)$ is a fuzzy metric space. We call the fuzzy metric $M_{d}$ induced by the metric $d$ the standard fuzzy metric. Note that every standard fuzzy metric is triangular.

Definition 1.11 Let $(X, M, *)$ is a fuzzy metric space and $t>0$. (i): A subset $A \subseteq X$ is said to be closed if for each convergent sequence $\left\{x_{n}\right\}$ with $x_{n} \in A$ and $x_{n} \rightarrow x$ as $n \rightarrow \infty$, we have $x \in A$.

(ii): $A \subseteq X$ is said to be compact if each sequence in $A$ has a convergent subsequence.

Throughout the article, let $\mathcal{P}(X), \mathcal{C}(X)$, and $\mathcal{K}(X)$ denote the set of all non-empty subsets, the set of all non-empty closed subsets, and the set of all non-empty compact subsets of $X$, respectively.

Definition 1.12 Let $X$ be a non-empty set. A point $x \in X$ is called a coincidence point of the mappings $F: X \rightarrow \mathcal{P}(X)$ and $f: X \rightarrow X$ if $f x \in F x$. Point $x \in X$ is called a fixed point of the mappings $F: X \rightarrow \mathcal{P}(X)$ if $f x \in F x$. 
Theorem 1.13 [16] Let $(X, M, *)$ be a fuzzy metric space. For each $A, B \in \mathcal{K}(X)$ and $t>0$ define

$$
H_{M}(A, B, t)=\min \left\{\inf _{a \in A} M(a, B, t), \inf _{b \in B} M(A, b, t)\right\}
$$

where $M(a, B, t):=\sup \{M(a, b, t): b \in B\}$. Then the 3-tuple $\left(\mathcal{K}(X), H_{M}, *\right)$ is a fuzzy metric space.

The fuzzy metric $\left(H_{M}, *\right)$ will be called the Hausdorff fuzzy metric of $(M, *)$ on $\mathcal{K}(X)$.

Lemma 1.14 [16] Let $(X, M, *)$ be a fuzzy metric space. Then, for each $a \in X, B \in \mathcal{K}(X)$ and $t>0$, there is $b_{0} \in B$ such that

$$
M(a, B, t)=M\left(a, b_{0}, t\right) .
$$

\section{Main results}

Throughout this section, $*$ denotes a continuous $t$-norm and $\mathcal{T}$ the set of all continuous real-valued mappings $T:[0,1]^{6} \rightarrow R$ satisfying the following properties:

$\mathcal{T}_{1}: T\left(t_{1}, t_{2}, \ldots, t_{6}\right)$ is non-increasing in $t_{2}, \ldots, t_{6}$.

$\mathcal{T}_{2}$ : If there exists $k \in(0,1)$ such that for each $t>0$, we have

$$
T\left(w(k t), v(t), v(t), u(t), u\left(\frac{t}{2}\right) * v\left(\frac{t}{2}\right), 1\right) \geq 1,
$$

where $u, v, w:(0, \infty) \rightarrow[0,1]$ are non-decreasing functions with $u(t), v(t), w(t) \in(0,1]$, then $w(k t) \geq v(t)$.

$\mathcal{T}_{3}$ : For each $t>0$ and some $k \in(0,1)$, the condition

$$
T(w(k t), 1,1, v(t), v(t), 1) \geq 1,
$$

implies $w(k t) \geq v(t)$.

Now we give our main result.

Theorem 2.1 Let $(X, M, *)$ be a complete fuzzy metric space with Hadžić-type $t$-norm * such that $M(x, y, t) \rightarrow 1$ as $t \rightarrow \infty$, for all $x, y \in X$. Let $\preceq$ be a partial order defined on $X$. Let $F: X \rightarrow \mathcal{K}(X)$ be a set-valued mapping with non-empty compact values and $f: X \rightarrow X$ a mapping such that $f(X)$ is closed and for some $T \in \mathcal{T}$ and all comparable elements $x, y \in X$, and $t>0$, we have

$$
\begin{aligned}
& T\left(H_{M}(F x, F y, k t), M(f x, f y, t), M(f x, F x, t), M(f y, F y, t),\right. \\
& \quad M(f x, F y, t), M(f y, F x, t)) \geq 1 .
\end{aligned}
$$

Also suppose that the following conditions are satisfied:

(i) $F(X) \subseteq f(X)$,

(ii) $f y \in F(x)$ implies $x \preceq y$,

(iii) if $y_{n} \in F\left(x_{n}\right)$ is a sequence such that $y_{n} \rightarrow y=f x$, then $x_{n} \preceq x$ for all $n$. 
Then $F$ and $f$ have a coincidence point, that is, there exists $x \in X$ such that $f x \in F(x)$.

Proof Let $t>0$ be fixed and $x_{0} \in X$. By using (i) and (ii), there exists $x_{1} \in X$ such that $x_{0} \preceq x_{1}$ and $y_{0}=f x_{1} \in F x_{0}$. Now from (i), (ii), and by Lemma 1.14, for $x_{1} \in X$ there is $x_{2} \in X$ such that $x_{1} \preceq x_{2}$ and $y_{1}=f x_{2} \in F x_{1}$ with

$$
M\left(y_{0}, F x_{1}, t\right)=M\left(y_{0}, y_{1}, t\right)
$$

thus

$$
H_{M}\left(F x_{0}, F x_{1}, t\right) \leq \sup _{y_{1} \in F x_{1}} M\left(y_{0}, y_{1}, t\right)=M\left(y_{0}, F x_{1}, t\right)=M\left(y_{0}, y_{1}, t\right) .
$$

On the other hand by $x=x_{0}$ and $y=x_{1}$ in (2.1), we have

$$
\begin{aligned}
& T\left(H_{M}\left(F x_{0}, F x_{1}, k t\right), M\left(f x_{0}, f x_{1}, t\right), M\left(f x_{0}, F x_{0}, t\right), M\left(f x_{1}, F x_{1}, t\right),\right. \\
& \left.\quad M\left(f x_{0}, F x_{1}, t\right), M\left(f x_{1}, F x_{0}, t\right)\right) \geq 1 .
\end{aligned}
$$

Now since $M\left(f x_{0}, F x_{0}, t\right) \geq M\left(f x_{0}, y_{0}, t\right), M\left(f x_{1}, F x_{1}, t\right) \geq M\left(y_{0}, y_{1}, t\right)$, also

$$
M\left(f x_{0}, F x_{1}, t\right) \geq M\left(f x_{0}, y_{1}, t\right) \geq M\left(f x_{0}, y_{0}, \frac{t}{2}\right) * M\left(y_{0}, y_{1}, \frac{t}{2}\right),
$$

and $M\left(f x_{1}, F x_{0}, t\right) \geq M\left(y_{0}, y_{0}, t\right)=1$, and by using $\mathcal{T}_{1}$, we get

$$
\begin{aligned}
& T\left(H_{M}\left(F x_{0}, F x_{1}, k t\right), M\left(f x_{0}, f x_{1}, t\right), M\left(f x_{0}, y_{0}, t\right), M\left(f x_{1}, y_{1}, t\right),\right. \\
& \left.\quad M\left(f x_{0}, y_{0}, \frac{t}{2}\right) * M\left(y_{0}, y_{1}, \frac{t}{2}\right), 1\right) \geq 1 .
\end{aligned}
$$

This means that

$$
T\left(w(k t), v(t), v(t), u(t), u\left(\frac{t}{2}\right) * v\left(\frac{t}{2}\right), 1\right) \geq 1,
$$

where $w(t)=H_{M}\left(F x_{0}, F x_{1}, t\right), v(t)=M\left(f x_{0}, f x_{1}, t\right), u(t)=M\left(f x_{1}, y_{1}, t\right)$, then from $\mathcal{T}_{2}$, we have $(w(k t) \geq v(t))$

$$
H_{M}\left(F x_{0}, F x_{1}, k t\right) \geq M\left(f x_{0}, f x_{1}, t\right)=M\left(f x_{0}, y_{0}, t\right)
$$

hence by (2.2), we obtain

$$
M\left(y_{0}, y_{1}, t\right) \geq M\left(f x_{0}, y_{0}, \frac{t}{k}\right) .
$$

Again by (i), (ii), and by Lemma 1.14, there exists $x_{3} \in X$ such that $x_{2} \preceq x_{3}$ with $y_{2}=f x_{3} \in$ $F x_{2}$ that satisfying in

$$
H_{M}\left(F x_{1}, F x_{2}, t\right) \leq M\left(y_{1}, F x_{2}, t\right)=M\left(y_{1}, y_{2}, t\right) .
$$


Since $x_{1} \preceq x_{2}$ thus by replacing $x=x_{1}$ and $y=x_{2}$ in (2.1) and from $\mathcal{T}_{1}$, we obtain

$$
\begin{aligned}
& T\left(H_{M}\left(F x_{1}, F x_{2}, k t\right), M\left(y_{0}, y_{1}, t\right), M\left(y_{0}, y_{1}, t\right), M\left(y_{1}, y_{2}, t\right),\right. \\
& \left.M\left(y_{0}, y_{1}, \frac{t}{2}\right) * M\left(y_{1}, y_{2}, \frac{t}{2}\right), 1\right) \geq 1
\end{aligned}
$$

Now by $w(t)=H_{M}\left(F x_{1}, F x_{2}, t\right), v(t)=M\left(y_{0}, y_{1}, t\right), u(t)=M\left(y_{1}, y_{2}, t\right)$, the property $\mathcal{T}_{2}$ implies

$$
H_{M}\left(F x_{1}, F x_{2}, k t\right) \geq M\left(y_{0}, y_{1}, t\right)
$$

thus from (2.3), we get

$$
M\left(y_{1}, y_{2}, t\right) \geq M\left(y_{0}, y_{1}, \frac{t}{k}\right)
$$

Repeatedly, there exists $x_{4} \in X$ with $x_{3} \preceq x_{4}$ such that $y_{3}=f x_{4} \in F x_{3}$ and $H_{M}\left(F x_{2}, F x_{3}, t\right) \leq$ $M\left(y_{2}, y_{3}, t\right)$, and

$$
H_{M}\left(F x_{2}, F x_{3}, k t\right) \geq M\left(y_{1}, y_{2}, t\right)
$$

therefore

$$
M\left(y_{2}, y_{3}, t\right) \geq M\left(y_{1}, y_{2}, \frac{t}{k}\right) \geq M\left(y_{0}, y_{1}, \frac{t}{k^{2}}\right) \geq M\left(f x_{0}, y_{0}, \frac{t}{k^{3}}\right) .
$$

Continuing the process, we can have a sequence $\left\{x_{n}\right\}$ in $X$ with $x_{n} \preceq x_{n+1}$ such that, for $n \geq 0, y_{n}=f x_{n+1} \in F x_{n}$, and

$$
M\left(y_{n}, y_{n+1}, t\right) \geq M\left(y_{n-1}, y_{n}, \frac{t}{k}\right)
$$

and

$$
M\left(y_{n}, y_{n+1}, t\right) \geq M\left(y_{0}, y_{1}, \frac{t}{k^{n}}\right) \geq M\left(f x_{0}, y_{0}, \frac{t}{k^{n+1}}\right)
$$

From (2.4), we conclude that, for each $i \geq 1$,

$$
M\left(y_{n+i}, y_{n+i+1}, t\right) \geq M\left(y_{n}, y_{n+1}, \frac{t}{k^{i}}\right)
$$

Next, we prove that the sequence $y_{n}$ is Cauchy. Suppose that $\delta>0$ and $\epsilon \in(0,1)$ are given. Then, by Lemma 1.6 and (FM-4), for all $m>n$,

$$
\begin{aligned}
M\left(y_{n}, y_{m}, \delta\right) \geq & M\left(y_{n}, y_{m}, \delta(1-k)\left(1+k+\cdots+k^{m-n-1}\right)\right) \\
\geq & M\left(y_{n}, y_{n+1}, \delta(1-k)\right) * M\left(y_{n+1}, y_{n+2}, \delta k(1-k)\right) * \cdots \\
& * M\left(y_{m-1}, y_{m}, \delta k^{m-n-1}(1-k)\right) .
\end{aligned}
$$


On the other hand, putting $t=\delta k^{i}(1-k)$ in (2.6), for all $n \geq 0, i \geq 1$, we get

$$
M\left(y_{n+i}, y_{n+i+1}, \delta k^{i}(1-k)\right) \geq M\left(y_{n}, y_{n+1}, \delta(1-k)\right)
$$

Then by replacing the above inequality in (2.7), we obtain, for all $m>n$,

$$
\begin{aligned}
M\left(y_{n}, y_{m}, \delta\right) \geq & M\left(y_{n}, y_{n+1}, \delta(1-k)\right) * M\left(y_{n+1}, y_{n+2}, \delta(1-k)\right) * \cdots \\
& * M\left(y_{m-1}, y_{m}, \delta(1-k)\right) \\
= & *^{(m-n)} M\left(y_{n}, y_{n+1}, \delta(1-k)\right) .
\end{aligned}
$$

By hypothesis, $*$ is a $t$-norm of Hadžić-type, and there exists $\eta \in(0,1)$ such that for all $m>n$,

$$
1 \geq s>\eta \quad \Rightarrow \quad *^{(m-n)}(s)>1-\epsilon .
$$

By $M\left(f x_{0}, y_{0}, t\right) \rightarrow 1$ as $t \rightarrow \infty$, there exists $n_{0}$ such that, for all $n \geq n_{0}$,

$$
M\left(f x_{0}, y_{0}, \frac{\delta(1-k)}{k^{n+1}}\right)>\eta
$$

From (2.5) and the above inequality, we have

$$
M\left(y_{n}, y_{n+1}, \delta(1-k)\right)>\eta
$$

therefore, (2.8) and (2.9) imply that, for all $n \geq n_{0}$ and each $m>n$,

$$
M\left(y_{n}, y_{m}, \delta\right)>1-\epsilon
$$

This shows that $\left\{y_{n}\right\}$ is a Cauchy sequence. Since $X$ is complete, there exists some $y \in X$ such that

$$
\lim _{n \rightarrow \infty} y_{n}=\lim _{n \rightarrow \infty} f\left(x_{n+1}\right)=y \in \lim _{n \rightarrow \infty} F\left(x_{n}\right) .
$$

Now, since $f(X)$ is closed, there exists $\bar{x} \in X$ such that $y=f \bar{x} \in f(X)$. Also (ii) implies that $x_{n} \preceq \bar{x}$ for any $n$. Thus from (2.1), we have

$$
\begin{aligned}
& T\left(H_{M}\left(F x_{n}, F \bar{x}, k t\right), M\left(f x_{n}, f \bar{x}, t\right), M\left(f x_{n}, F x_{n}, t\right), M(f \bar{x}, F \bar{x}, t),\right. \\
& \left.\quad M\left(f x_{n}, F \bar{x}, t\right), M\left(f \bar{x}, F x_{n}, t\right)\right) \geq 1 .
\end{aligned}
$$

By taking the limit as $n \rightarrow \infty$, by the continuity of $T$, and from Lemma 1.8 , we get

$$
T\left(\lim _{n \rightarrow \infty} H_{M}\left(F x_{n}, F \bar{x}, k t\right), 1,1, M(y, F \bar{x}, t), M(y, F \bar{x}, t), 1\right) \geq 1 .
$$

Now by using $\mathcal{T}_{3}$, we have

$$
\lim _{n \rightarrow \infty} H_{M}\left(F x_{n}, F \bar{x}, k t\right) \geq M(y, F \bar{x}, t),
$$


on the other hand $H_{M}\left(F x_{n}, F \bar{x}, k t\right) \leq M\left(y_{n}, F \bar{x}, k t\right)$, so

$$
M(y, F \bar{x}, k t) \geq \lim _{n \rightarrow \infty} H_{M}\left(F x_{n}, F \bar{x}, k t\right) \geq M(y, F \bar{x}, t) .
$$

It follows that $M(y, F \bar{x}, t)=1$ for each $t>0$. Now since $F \bar{x}$ is closed (note that $F \bar{x}$ is compact), we get $f \bar{x}=y \in F \bar{x}$, thus $\bar{x}$ is a coincidence point of $F$ and $f$. The proof is complete.

Remark 2.2 In Theorem 2.1, we proved that the sequence $y_{n}$ is Cauchy; one can replace the condition ' $*$ is Hadžić-type $t$-norm and $M(x, y, t) \rightarrow 1$ as $t \rightarrow \infty$, for all $x, y \in X$ ' with the following: ' $\lim _{n \rightarrow \infty} *_{i=n}^{\infty} M\left(x, y, t h^{i}\right)=1$ for each $h>1$ '. To see this, choose some $q>1$ and $n \in \mathbb{N}$ such that $k q<1$ and $\sum_{i=n_{1}}^{\infty} \frac{1}{q^{i}} \leq 1$. Then from (FM-4) and (2.5), for every $m>n \geq n_{1}$, we have

$$
\begin{aligned}
& M\left(y_{n}, y_{m}, t\right) \\
& \quad \geq M\left(y_{n}, y_{m}, \sum_{i=n}^{m-1} \frac{1}{q^{i}} t\right) \\
& \geq M\left(y_{n}, y_{n+1}, \frac{1}{q^{n}} t\right) * M\left(y_{n+1}, y_{n+2}, \frac{1}{q^{n+1}} t\right) * \cdots * M\left(y_{m-1}, y_{m}, \frac{1}{q^{m-1}} t\right) \\
& \geq M\left(f x_{0}, y_{0}, \frac{1}{k^{n+1} q^{n}} t\right) * M\left(f x_{0}, y_{0}, \frac{1}{k^{n+2} q^{n+1}} t\right) * \cdots * M\left(f x_{0}, y_{0}, \frac{1}{k^{m} q^{m-1}} t\right) \\
& \geq M\left(f x_{0}, y_{0}, \frac{1}{(k q)^{n+1}} t\right) * M\left(f x_{0}, y_{0}, \frac{1}{(k q)^{n+2}} t\right) * \cdots * M\left(f x_{0}, y_{0}, \frac{1}{(k q)^{m}} t\right) \\
& \geq \underset{\substack{* \\
i=n}}{*} M\left(f x_{0}, y_{0}, \frac{1}{(k q)^{i+1}} t\right)>1-\epsilon .
\end{aligned}
$$

Thus, $\left\{y_{n}\right\}$ is a Cauchy sequence. Then we have the following theorem.

Theorem 2.3 Let $(X, M, *)$ be a complete fuzzy metric space and suppose for each $h>1$, $\lim _{n \rightarrow \infty} *_{i=n}^{\infty} M\left(x, y, t h^{i}\right)=1$. Let $\preceq$ be a partial order defined on $X$. Let $F: X \rightarrow \mathcal{K}(X)$ be a set-valued mapping with non-empty compact values and $f: X \rightarrow X$ a mapping such that $f(X)$ is closed and for some $T \in \mathcal{T}$ and all comparable elements $x, y \in X$, and $t>0$, we have

$$
\begin{aligned}
& T\left(H_{M}(F x, F y, k t), M(f x, f y, t), M(f x, F x, t), M(f y, F y, t),\right. \\
& \quad M(f x, F y, t), M(f y, F x, t)) \geq 1 .
\end{aligned}
$$

Also suppose that the following conditions are satisfied:

(i) $F(X) \subseteq f(X)$,

(ii) $f y \in F(x)$ implies $x \preceq y$,

(iii) if $y_{n} \in F\left(x_{n}\right)$ is a sequence such that $y_{n} \rightarrow y=f x$, then $x_{n} \preceq x$ for all $n$.

Then $F$ and $f$ have a coincidence point, that is, there exists $x \in X$ such that $f x \in F(x)$.

If in Theorem 2.1 and 2.3 we put $T\left(u_{1}, \ldots, u_{6}\right):=\frac{u_{1}(k t)}{u_{2}(t)}$, where $k \in(0,1)$, then we have the following corollaries.

Corollary 2.4 Let $(X, M, *)$ be a complete fuzzy metric space with Hadžić-type $t$-norm * such that $M(x, y, t) \rightarrow 1$ as $t \rightarrow \infty$, for all $x, y \in X$. Let $\preceq$ be a partial order defined on $X$. 
Let $F: X \rightarrow \mathcal{K}(X)$ be a set-valued mapping with non-empty compact values and $f: X \rightarrow X$ a mapping such that $f(X)$ be closed and for all comparable elements $x, y \in X$, and $t>0$, we have

$$
H_{M}(F x, F y, k t) \geq M(f x, f y, t) .
$$

Also suppose that the following conditions are satisfied:

(i) $F(X) \subseteq f(X)$,

(ii) $f y \in F(x)$ implies $x \preceq y$,

(iii) if $y_{n} \in F\left(x_{n}\right)$ is a sequence such that $y_{n} \rightarrow y=f x$, then $x_{n} \preceq x$ for all $n$.

Then there exists $x \in X$ such that $f x \in F(x)$.

Corollary 2.5 Let $(X, M, *)$ be a complete fuzzy metric space and suppose for each $h>1$, $\lim _{n \rightarrow \infty} *_{i=n}^{\infty} M\left(x, y, t h^{i}\right)=1$. Let $\preceq$ be a partial order defined on $X$. Let $F: X \rightarrow \mathcal{K}(X)$ be a set-valued mapping with non-empty compact values and $f: X \rightarrow X$ a mapping such that $f(X)$ be closed and for all comparable elements $x, y \in X$, and $t>0$, we have

$$
H_{M}(F x, F y, k t) \geq M(f x, f y, t) .
$$

Also suppose that the following conditions are satisfied:

(i) $F(X) \subseteq f(X)$,

(ii) $f y \in F(x)$ implies $x \preceq y$,

(iii) if $y_{n} \in F\left(x_{n}\right)$ is a sequence such that $y_{n} \rightarrow y=f x$, then $x_{n} \preceq x$ for all $n$.

Then there exists $x \in X$ such that $f x \in F(x)$.

Putting $f=I$ (the identity mapping) in Corollary 2.4 and 2.5 , we get the following corollaries.

Corollary 2.6 Let $(X, M, *)$ be a complete fuzzy metric space with Hadžić-type $t$-norm * such that $M(x, y, t) \rightarrow 1$ as $t \rightarrow \infty$, for some $x_{0} \in X$ and $x_{1} \in F x_{0}$. Let $\preceq$ be a partial order defined on $X$. Let $F: X \rightarrow \mathcal{K}(X)$ be a set-valued mapping with non-empty compact values for all comparable elements $x, y \in X$, and $t>0$, we have

$$
H_{M}(F x, F y, k t) \geq M(x, y, t) .
$$

Also suppose that the following conditions are satisfied:

(i) $y \in F(x)$ implies $x \preceq y$,

(ii) if $y_{n} \in F\left(x_{n}\right)$ is a sequence such that $y_{n} \rightarrow x$, then $x_{n} \preceq x$ for all $n$.

Then $F$ has a fixed point.

Corollary 2.7 Let $(X, M, *)$ be a complete fuzzy metric space and suppose for each $h>1$, $\lim _{n \rightarrow \infty} *_{i=n}^{\infty} M\left(x, y, t h^{i}\right)=1$ for some $x_{0} \in X$ and $x_{1} \in F x_{0}$. Let $\preceq$ be a partial order defined on $X$. Let $F: X \rightarrow \mathcal{K}(X)$ be a set-valued mapping with non-empty compact values for all comparable elements $x, y \in X$, and $t>0$, we have

$$
H_{M}(F x, F y, k t) \geq M(x, y, t) .
$$

Also suppose that the following conditions are satisfied: 
(i) $y \in F(x)$ implies $x \preceq y$,

(ii) if $y_{n} \in F\left(x_{n}\right)$ is a sequence such that $y_{n} \rightarrow x$, then $x_{n} \preceq x$ for all $n$.

Then $F$ has a fixed point.

Remark 2.8 Note that we assumed the implicit relation (2.1) only for the comparable elements of the partially ordered fuzzy metric space.

Remark 2.9 Corollary 2.7 improves and generalizes the mentioned result of Gregori and Sapena (see Theorem 4.8 of [8]) for set-valued mappings in complete partially ordered fuzzy metric spaces.

In continuation, in the spirit of Miheț [19], we introduce the notion of a set-valued fuzzy order $\psi$-contraction of $(\epsilon, \lambda)$-type mappings and give a fixed point theorem in partially ordered fuzzy metric spaces.

Definition 2.10 Let $(X, M, *)$ be a fuzzy metric space and $\psi:(0,1) \rightarrow(0,1)$. A mapping $F: X \rightarrow \mathcal{C}(X)$ is a set-valued fuzzy order $\psi$-contraction of $(\epsilon, \lambda)$-type if the following implication holds:

$$
M(x, y, \epsilon)>1-\lambda \quad \Rightarrow \quad \forall p \in F x \exists q \in F y ; \quad M(p, q, \epsilon)>1-\psi(\lambda),
$$

for every $\epsilon>0, \lambda \in(0,1)$ and all comparable elements $x, y \in X$.

If $\psi(t)=\alpha t(t \in(0,1))$ for some $\alpha \in(0,1)$, then $F$ will be called a set-valued fuzzy order $\alpha$-contraction of $(\epsilon, \lambda)$-type.

Also note that if $\psi(t)<t$ for all $t \in(0,1)$, then every set-valued fuzzy order $\psi$-contraction of $(\epsilon, \lambda)$-type satisfies the relation

$$
\forall p \in F x \exists q \in F y ; \quad M(p, q, t) \geq M(x, y, t),
$$

for all comparable elements $x, y \in X$ and $t>0$. Indeed, if for some comparable $x, y \in X$ and $t>0$ there exists $p \in F x$ such that for all $q \in F y$, we have $M(p, q, t)<M(x, y, t)$; then there is $\lambda \in(0,1)$ such that $M(p, q, t)<1-\lambda<M(x, y, t)$, that is, $M(x, y, t)>1-\lambda$ and $M(p, q, t)<$ $1-\lambda<1-\psi(\lambda)$, which is a contradiction.

Example 2.11 Let $(X, M, *)$ be a fuzzy metric space. Let $F: Y \rightarrow \mathcal{C}(Y)$ be a set-valued mapping, where $Y \in \mathcal{C}(X)$. If there is $\alpha \in(0,1)$ such that

$$
\forall p \in F x \exists q \in F y ; \quad 1-M(p, q, t) \leq \alpha(1-M(x, y, t)),
$$

for all comparable elements $x, y \in X$ and $t>0$, then $F$ is a set-valued fuzzy order $\alpha$ contraction of $(\epsilon, \lambda)$-type. Indeed, if $M(x, y, \epsilon)>1-\lambda$, then for every comparable elements $x, y \in X$ and some $\alpha \in(0,1)$, we have

$$
\forall p \in F x \exists q \in F y ; \quad 1-M(p, q, \epsilon) \leq \alpha(1-M(x, y, \epsilon))<\alpha \lambda,
$$

thus $M(p, q, \epsilon)>1-\alpha \lambda$. 
Now we state our main theorem.

Theorem 2.12 Let $(X, M, *)$ be a complete fuzzy metric space with $\sup _{t<1} t * t=1$. $Y \in \mathcal{C}(X)$ and $F: Y \rightarrow \mathcal{C}(Y)$ be a set-valued fuzzy order $\psi$-contraction of $(\epsilon, \lambda)$-type, where $\lim _{n \rightarrow \infty} \psi^{(n)}(t)=0$ for all $t \in(0,1)$. Let ' $\leq$ ' be a partial order defined on $X$, and $\lim _{n \rightarrow \infty} *_{i=n}^{\infty}\left(1-\psi^{(i)}(\xi)\right)=1$ for all $\xi \in(0,1)$. Suppose that there exist $x_{0} \in Y$ and $x_{1} \in F x_{0}$ such that $M\left(x_{0}, x_{1}, 0_{+}\right)>0$ and the following two conditions hold:

(i) $y \in F(x)$ implies $x \preceq y$,

(ii) if $x_{n}$ is a sequence with $x_{n+1} \in F x_{n}$ and $x_{n} \rightarrow x$, then $x_{n} \preceq x$ for all $n$.

Then $F$ has a fixed point.

Proof Since there exist $x_{0} \in Y$ and $x_{1} \in F x_{0}$ such that $M\left(x_{0}, x_{1}, 0_{+}\right)>0$, we have $x_{0} \preceq x_{1}$ with $M\left(x_{0}, x_{1}, 0_{+}\right)>0$. We may suppose that $M\left(x_{0}, x_{1}, 0_{+}\right)<1$. For, if we assume the contrary, then $M\left(x_{0}, x_{1}, t\right)=1$ for all $t>0$, that is, $x_{0}=x_{1} \in F x_{0}$ and we have finished the proof. Therefore, for some $\delta_{1} \in(0,1)$ and every $t>0, \delta \in\left(\delta_{1}, 1\right)$, we have

$$
M\left(x_{0}, x_{1}, t\right) \geq M\left(x_{0}, x_{1}, 0_{+}\right)=1-\delta_{1}>1-\delta .
$$

Since $F$ is a set-valued fuzzy order $\psi$-contraction of $(\epsilon, \lambda)$-type mapping, there exists $x_{2} \in F x_{1}$ with $x_{1} \preceq x_{2}$ such that $M\left(x_{1}, x_{2}, t\right)>1-\psi(\delta)$. Repeating this argument, we get a sequence $\left\{x_{n}\right\}$ in $Y$ such that $x_{n+1} \in F x_{n}$ with $x_{n} \preceq x_{n+1}$ and such that

$$
M\left(x_{n}, x_{n+1}, t\right)>1-\psi^{n}(\delta) .
$$

Suppose that $\epsilon>0$ and $\lambda \in(0,1)$ are given. Since $\lim _{n \rightarrow \infty} *_{i=n}^{\infty}\left(1-\psi^{(i)}(\xi)\right)=1$ for all $\xi \in$ $(0,1)$, there exists $n_{0} \in \mathbb{N}$ such that for all $n \geq n_{0}$ and all $\xi \in(0,1)$, and we have

$$
\underset{i=n}{*}\left(1-\psi^{(i)}(\xi)\right)>1-\lambda
$$

Now by using (FM-4) and from (2.11)-(2.12), for all $m>n \geq n_{0}$, we get

$$
\begin{aligned}
& M\left(x_{n}, x_{m}, \epsilon\right) \\
& \quad \geq M\left(x_{n}, x_{n+1}, \frac{\epsilon}{m-n}\right) * M\left(x_{n+1}, x_{n+2}, \frac{\epsilon}{m-n}\right) * \cdots * M\left(x_{m-1}, x_{m}, \frac{\epsilon}{m-n}\right) \\
& \quad \geq\left(1-\psi^{(n)}(\delta)\right) *\left(1-\psi^{(n+1)}(\delta)\right) * \cdots *\left(1-\psi^{(m-1)}(\delta)\right) \\
& \quad \geq \underset{i=n}{*}\left(1-\psi^{(i)}(\delta)\right)>1-\lambda .
\end{aligned}
$$

This shows that $\left\{x_{n}\right\}$ is a Cauchy sequence. Since $X$ is complete, $\left\{x_{n}\right\}$ converges to some $\bar{x} \in$ $X$, that is, $\lim _{n \rightarrow \infty} M\left(x_{n}, \bar{x}, t\right)=1$. Now we prove that $\bar{x} \in F \bar{x}$. But $F \bar{x}=\overline{F \bar{x}}$; then it is enough to show that for every $\epsilon^{\prime}>0$ and $\lambda^{\prime} \in(0,1)$ there exists $z \in F \bar{x}$ such that $M\left(\bar{x}, z, \epsilon^{\prime}\right)>1-\lambda^{\prime}$.

Let $\epsilon^{\prime}>0$ and $\lambda^{\prime} \in(0,1)$ be arbitrary. From $\sup _{t<1} t * t=1$, it follows that there exists $\lambda_{1}\left(\lambda^{\prime}\right) \in(0,1)$ such that

$$
\left(1-\lambda_{1}\right) *\left(1-\lambda_{1}\right)>1-\lambda^{\prime} .
$$


Also for $\lambda_{1}$ there are $\lambda_{2} \in(0,1)$ such that

$$
\left(1-\lambda_{2}\right) *\left(1-\lambda_{2}\right)>1-\lambda_{1}
$$

Now put $\lambda_{3}=\min \left\{\lambda_{1}, \lambda_{2}\right\}$. We prove that there exists $\mu \in(0,1)$ such that $\psi(\mu)<\lambda_{3}$. For, if $\psi(t) \geq \lambda_{3}$ for every $t \in(0,1)$, then $\psi^{n}(t) \geq \lambda_{3}$ for every $n \in \mathbb{N}$ and every $t \in(0,1)$, therefore $*_{i=n}^{\infty}\left(1-\psi^{(i)}(\xi)\right) \leq *_{i=n}^{\infty}\left(1-\lambda_{3}\right) \leq 1-\lambda_{3}$ for all $n \in \mathbb{N}$, which means that $1=\lim _{n \rightarrow \infty} *_{i=n}^{\infty}(1-$ $\left.\psi^{(i)}(\xi)\right) \leq 1-\lambda_{3}<1$, and this is a contradiction.

Since $\lim _{n \rightarrow \infty} M\left(x_{n}, \bar{x}, t\right)=1$ for all $t>0$, there exists $n_{1} \in \mathbb{N}$ such that for all $n \geq n_{1}$, and we have $M\left(x_{n}, \bar{x}, \frac{\epsilon^{\prime}}{3}\right)>1-\mu$; thus, since $x_{n} \preceq \bar{x}$ and by using (2.10), there exists $z \in F \bar{x}$ such that

$$
M\left(x_{n+1}, z, \frac{\epsilon^{\prime}}{3}\right)>1-\psi(\mu)>1-\lambda_{3} .
$$

On the other hand $\lim _{n \rightarrow \infty} \psi^{(n)}(t)=0$ for every $t \in(0,1)$. Therefore $(2.11)$ implies the existence of the element $n_{2} \in \mathbb{N}$ such that for all $n \geq n_{2}$, we have

$$
M\left(x_{n}, x_{n+1}, \frac{\epsilon^{\prime}}{3}\right)>1-\lambda_{3} .
$$

Also since $\lim _{n \rightarrow \infty} x_{n}=\bar{x}$, there exists $n_{3} \in \mathbb{N}$ such that for all $n \geq n_{3}$,

$$
M\left(x_{n}, \bar{x}, \frac{\epsilon^{\prime}}{3}\right)>1-\lambda_{3} .
$$

Now if $n \geq \max \left\{n_{1}, n_{2}, n_{3}\right\}$, then by (2.13)-(2.17), we get

$$
\begin{aligned}
M\left(\bar{x}, z, \epsilon^{\prime}\right) & \geq M\left(\bar{x}, x_{n}, \frac{\epsilon^{\prime}}{3}\right) * M\left(x_{n}, x_{n+1}, \frac{\epsilon^{\prime}}{3}\right) * M\left(x_{n+1}, z, \frac{\epsilon^{\prime}}{3}\right) \\
& >\left(1-\lambda_{3}\right) *\left(1-\lambda_{3}\right) *\left(1-\lambda_{3}\right)>1-\lambda^{\prime} .
\end{aligned}
$$

Hence $\bar{x} \in F \bar{x}=\overline{F \bar{x}}$, consequently $\bar{x}$ is a fixed point of $F$. The theorem is proved.

Corollary 2.13 Let $(X, M, *)$ be a complete fuzzy metric space with Lukasiewicz $t$-norm and '`' be a partial order defined on $X$. Let $Y \in \mathcal{C}(X)$ and $F: Y \rightarrow \mathcal{C}(Y)$ be a set-valued mapping with the property that there is $\alpha \in(0,1)$ such that

$$
\forall p \in F x \exists q \in F y ; \quad 1-M(p, q, t) \leq \alpha(1-M(x, y, t))
$$

for all comparable elements $x, y \in X$ and $t>0$, and the following conditions hold:

(i) $y \in F(x)$ implies $x \preceq y$,

(ii) if $x_{n}$ is a sequence with $x_{n+1} \in F x_{n}$ and $x_{n} \rightarrow x$, then $x_{n} \preceq x$ for all $n$.

Then $F$ has a fixed point.

Proof By using Definition 1.2, $\sup _{t<1} t * t=1$. Also, from Example 2.11 it follows that $F$ is a set-valued fuzzy order $\psi$-contraction of $(\epsilon, \lambda)$-type with $\psi(t)=\alpha t$. Since, for all $\lambda \in(0,1)$, 
$\sum_{i=1}^{\infty} \psi^{(i)}(\lambda)=\sum_{i=1}^{\infty} \alpha^{i} \lambda<\infty$, from Proposition 1.3, we have $\lim _{n \rightarrow \infty} *_{i=n}^{\infty}\left(1-\psi^{(i)}(\lambda)\right)=1$.

Next, since

$$
\forall p \in F x \exists q \in F y ; \quad M(p, q, t) \geq 1-\alpha+\alpha M(x, y, t) \geq 1-\alpha>0,
$$

for all comparable elements $x, y \in X$ and $t>0$, there exist $x_{0} \in Y$ and $x_{1} \in F x_{0}$ such that $M\left(x_{0}, x_{1}, 0_{+}\right)>0$. Consequently, by the preceding theorem, $F$ has a fixed point.

Corollary 2.14 Let $(X, M, *)$ be a complete fuzzy metric space with a continuous $g$ convergent $t$-norm and ' $\leq$ ' be a partial order defined on $X$. Let $Y \in \mathcal{C}(X)$ and $F: Y \rightarrow \mathcal{C}(Y)$ be a set-valued fuzzy order $\alpha$-contraction of $(\epsilon, \lambda)$-type. If there exist $x_{0} \in Y$ and $x_{1} \in F x_{0}$ such that $M\left(x_{0}, x_{1}, 0_{+}\right)>0$ and the following two conditions hold:

(i) $y \in F(x)$ implies $x \preceq y$,

(ii) if $x_{n}$ is a sequence with $x_{n+1} \in F x_{n}$ and $x_{n} \rightarrow x$, then $x_{n} \preceq x$ for all $n$.

Then $F$ has a fixed point.

Theorem 2.12 and Corollary 2.13 are, respectively, generalizations of the theorems of Mihet [19] and Tirado [20] to the set-valued case in partial ordered fuzzy metric spaces.

Now we introduce a definition and, by using it, we shall state fixed and common fixed point theorems in the partially ordered fuzzy metric space. Our results generalize and extend Theorems 4.1 and 4.2 of [21] to set-valued mappings in complete partially ordered fuzzy metric spaces.

Definition 2.15 Let $Y$ be a non-empty subset of fuzzy metric space $(X, M, *)$. Mapping $F: Y \rightarrow \mathcal{P}(X)$ is called fuzzy order $K$-set-valued mapping, if for all $x \in Y, u_{x} \in F x$, there exists $u_{y} \in F y$ with $u_{x} \preceq u_{y}$ such that

$$
\frac{1}{M\left(u_{x}, u_{y}, t\right)}-1 \leq k\left[\frac{1}{M\left(x, u_{x}, t\right)}-1+\frac{1}{M\left(y, u_{y}, t\right)}-1\right]
$$

for every $t>0$ and $y \in Y$ with $x \preceq y$ and some $k \in\left(0, \frac{1}{2}\right)$.

Theorem 2.16 Let $(X, M, *)$ be a complete fuzzy metric space, with $M$ triangular, and ' $\preceq$ 'a partial order on $X$. Let $Y \in \mathcal{C}(X)$ and $F: Y \rightarrow \mathcal{C}(Y)$ be a fuzzy order $K$-set-valued mapping. Also let there for some $x_{0} \in Y$ exist $x_{1} \in F x_{0}$ with $x_{0} \preceq x_{1}$, and the following condition is satisfied:

If $x_{n} \rightarrow x$ is a sequence in $Y$ whose consecutive terms are comparable, then $x_{n} \preceq x$, for all $n$.

Then $F$ has a fixed point in $X$.

Proof By the hypothesis, for $x_{0} \in Y$ there exists $x_{1} \in F x_{0}$ such that $x_{0} \preceq x_{1}$. Now because $F$ is a fuzzy order $K$-set-valued mapping, there exists $x_{2} \in F x_{1}$ such that $x_{1} \preceq x_{2}$ and

$$
\frac{1}{M\left(x_{1}, x_{2}, t\right)}-1 \leq k\left[\frac{1}{M\left(x_{0}, x_{1}, t\right)}-1+\frac{1}{M\left(x_{1}, x_{2}, t\right)}-1\right] \text {, }
$$

thus

$$
\frac{1}{M\left(x_{1}, x_{2}, t\right)}-1 \leq \frac{k}{1-k}\left[\frac{1}{M\left(x_{0}, x_{1}, t\right)}-1\right] .
$$


Then it follows by induction that

$$
\frac{1}{M\left(x_{n}, x_{n+1}, t\right)}-1 \leq\left(\frac{k}{1-k}\right)^{n}\left[\frac{1}{M\left(x_{0}, x_{1}, t\right)}-1\right]
$$

where $\left\{x_{n}\right\}$ is a sequence whose consecutive terms are comparable, that is, $x_{n+1} \in F x_{n}$. Now we prove that $\left\{x_{n}\right\}$ is a Cauchy sequence. By putting $\lambda=\frac{k}{1-k}$, and by (2.19), and since $M$ is triangular, we have for all $m>n$

$$
\begin{aligned}
\frac{1}{M\left(x_{n}, x_{m}, t\right)}-1 & \leq \sum_{i=0}^{m-n-1}\left[\frac{1}{M\left(x_{n+i}, x_{n+i+1}, t\right)}-1\right] \\
& \leq\left(\frac{1}{M\left(x_{0}, x_{1}, t\right)}-1\right) \sum_{i=n}^{m-1} \lambda^{i} \leq\left(\frac{1}{M\left(x_{0}, x_{1}, t\right)}-1\right) \frac{\lambda^{n}}{1-\lambda}
\end{aligned}
$$

For each $t>0$ and each $\epsilon \in(0,1)$, we can choose a sufficiently large $n_{0} \in \mathbb{N}$ such that

$$
\left(\frac{1}{M\left(x_{0}, x_{1}, t\right)}-1\right) \frac{\lambda^{n_{0}}}{1-\lambda}<\frac{1}{1-\epsilon}-1
$$

Thus from (2.20) and (2.21), $M\left(x_{n}, x_{m}, t\right)>1-\epsilon$, for all $m, n>n_{0}$ and $t>0$. This shows that the sequence $\left\{x_{n}\right\}$ is Cauchy, and, since $X$ is complete, it converges to a point $x \in X$. But $Y$ is closed, thus $x \in Y$ and also by using the hypothesis $x_{n} \preceq x$. Now we show that $x \in F x$. From $x_{n} \in F x_{n-1}$, and $x_{n-1} \preceq x$ for all $n$, since $F$ is a fuzzy order $K$-set-valued mapping, there exists $u_{n} \in F x$ such that $x_{n} \preceq u_{n}$, and

$$
\frac{1}{M\left(x_{n}, u_{n}, t\right)}-1 \leq k\left[\frac{1}{M\left(x_{n-1}, x_{n}, t\right)}-1+\frac{1}{M\left(x, u_{n}, t\right)}-1\right]
$$

Now since $M$ is triangular, by using (2.22), we get

$$
\frac{1}{M\left(x, u_{n}, t\right)}-1 \leq \frac{1}{1-k}\left[\frac{1}{M\left(x, x_{n}, t\right)}-1+\frac{1}{M\left(x_{n-1}, x_{n}, t\right)}-1\right]
$$

and so, letting $n \rightarrow \infty, u_{n} \rightarrow x$. Consequently, since $F x$ is closed, we have $x \in F x$. Then $F$ has a fixed point.

From the above theorem we can immediately obtain the following generalization for getting a common fixed point.

Theorem 2.17 Let $(X, M, *)$ be a complete fuzzy metric space, with $M$ triangular, and ' a partial order on $X$. Let $Y \in \mathcal{C}(X)$ and, for every $n \in \mathbb{N}, F_{n}: Y \rightarrow \mathcal{C}(Y)$ be a sequence of mappings such that, for every two mappings $F_{i}, F_{j}$ and for all $x \in Y, u_{x} \in F_{i}(x)$, there exists $u_{y} \in F_{j}(y)$ with $u_{x} \preceq u_{y}$ such that

$$
\frac{1}{M\left(u_{x}, u_{y}, t\right)}-1 \leq k\left[\frac{1}{M\left(x, u_{x}, t\right)}-1+\frac{1}{M\left(y, u_{y}, t\right)}-1\right]
$$

for every $t>0$ and $y \in Y$ with $x \preceq y$ and some $k \in\left(0, \frac{1}{2}\right)$. Also let there exist, for some $x_{0} \in Y$, $x_{1} \in F_{1} x_{0}$ with $x_{0} \preceq x_{1}$, and the following condition be satisfied: 
If $x_{n} \rightarrow x$ is a sequence in $Y$ whose consecutive terms are comparable, then $x_{n} \preceq x$, for all $n$.

Then there exists $x \in Y$ such that $x \in \bigcap F_{n} x$, that is, $\left\{F_{n}\right\}$ has a common fixed point.

Proof We can find $x_{2} \in F_{2} x_{1}$ such that $x_{1} \preceq x_{2}$ and that

$$
\frac{1}{M\left(x_{1}, x_{2}, t\right)}-1 \leq \frac{k}{1-k}\left[\frac{1}{M\left(x_{0}, x_{1}, t\right)}-1\right] .
$$

Also for $x_{2}$ there exists $x_{3} \in F_{3} x_{2}$ with $x_{2} \preceq x_{3}$ and such that

$$
\frac{1}{M\left(x_{2}, x_{3}, t\right)}-1 \leq \frac{k}{1-k}\left[\frac{1}{M\left(x_{0}, x_{1}, t\right)}-1\right] .
$$

By continuing this process, we get

$$
\frac{1}{M\left(x_{n}, x_{n+1}, t\right)}-1 \leq\left(\frac{k}{1-k}\right)^{n}\left[\frac{1}{M\left(x_{0}, x_{1}, t\right)}-1\right],
$$

where $\left\{x_{n}\right\}$ is a sequence with $x_{n+1} \in F_{n+1} x_{n}$. Now similar to the proof of the preceding theorem, we can prove that $\left\{x_{n}\right\}$ is a Cauchy sequence and by the completeness of $X$ it follows that $\left\{x_{n}\right\}$ converges to some $x \in X$. Furthermore, $x \in Y$ and $x_{n} \preceq x$. Now suppose that $F_{N}$ is any arbitrary member of $F_{n}$. Since $x_{n} \in F_{n} x_{n-1}, x_{n-1} \preceq x$ for all $n$, and by the hypothesis, there exists $u_{n} \in F_{N} x$ such that $x_{n} \preceq u_{n}$, and

$$
\frac{1}{M\left(x_{n}, u_{n}, t\right)}-1 \leq k\left[\frac{1}{M\left(x_{n-1}, x_{n}, t\right)}-1+\frac{1}{M\left(x, u_{n}, t\right)}-1\right],
$$

thus

$$
\frac{1}{M\left(x, u_{n}, t\right)}-1 \leq \frac{1}{1-k}\left[\frac{1}{M\left(x, x_{n}, t\right)}-1+\frac{1}{M\left(x_{n-1}, x_{n}, t\right)}-1\right] .
$$

Next by the letting $n \rightarrow \infty$, we get $u_{n} \rightarrow x$, and then $x \in F_{N} x$. As $F_{N}$ is an arbitrary member of $F_{n}, x \in \bigcap F_{n} x$, and $x$ is a common fixed point of $\left\{F_{n}\right\}$. The theorem is proved.

Example 2.18 Let $X=[0, \infty)$ with $t$-norm defined $a * b=\min \{a, b\}$ for all $a, b \in[0,1]$ and $M(x, y, t)=\frac{t}{t+|x-y|}$, for all $x, y \in X$ and $t>0$. Then $(X, M, *)$ is a complete fuzzy metric space. Let the natural ordering $\leq$ of the numbers as the partial ordering $\preceq$. Define $Y=[0,1]$ and $F: Y \rightarrow \mathcal{C}(Y)$ as $F x=\left\{z, \frac{x}{5}\right\}$ for each $0 \leq x<\frac{1}{2}$, and $\left\{z, \frac{x}{4}\right\}$ for each $\frac{1}{2} \leq x \leq 1$, where $z \in Y$ is an arbitrary. If $x, y \in Y$ such that $x \preceq y$ and $u_{x}=z \in F x$, then there exists $u_{y}=z \in F y$ such that $u_{x} \preceq u_{y}$ and (2.18) is satisfied. Thus $F$ is a fuzzy order $K$-set-valued mapping. But if $u_{x} \neq z \in F x$, then three cases arise.

Case (i). If $0 \leq x \leq y<\frac{1}{2}$, then for every $t>0$

$$
\frac{1}{M\left(\frac{x}{5}, \frac{y}{5}, t\right)}-1=\frac{y-x}{5 t} \leq \frac{4}{9}\left[\frac{4(x+y)}{5 t}\right]=\frac{4}{9}\left[\frac{1}{M\left(x, \frac{x}{5}, t\right)}-1+\frac{1}{M\left(y, \frac{y}{5}, t\right)}-1\right] .
$$

Case (ii). If $\frac{1}{2} \leq x \leq y \leq 1$, then for every $t>0$

$$
\frac{1}{M\left(\frac{x}{4}, \frac{y}{4}, t\right)}-1=\frac{y-x}{4 t} \leq \frac{4}{9}\left[\frac{3(x+y)}{4 t}\right]=\frac{4}{9}\left[\frac{1}{M\left(x, \frac{x}{4}, t\right)}-1+\frac{1}{M\left(y, \frac{y}{4}, t\right)}-1\right] .
$$


Case (iii). If $0 \leq x<\frac{1}{2} \leq y \leq 1$, then for every $t>0$

$$
\frac{1}{M\left(\frac{x}{5}, \frac{y}{4}, t\right)}-1 \leq \frac{4}{9}\left[\frac{16 x+15 y}{20 t}\right]=\frac{4}{9}\left[\frac{1}{M\left(x, \frac{x}{5}, t\right)}-1+\frac{1}{M\left(y, \frac{y}{4}, t\right)}-1\right] .
$$

Hence $F$ is a fuzzy order $K$-set-valued mapping with $k=\frac{4}{9}<\frac{1}{2}$. Moreover, there exists $x_{0}=0$ (or $\left.x_{0}=z\right)$ with $x_{1}=0\left(x_{1}=z\right)$ such that $x_{0} \preceq x_{1}$. Thus all the hypotheses of Theorem 2.16 are satisfied and $x=0$ (or $x=z$ ) is the fixed point of $F$.

\section{Competing interests}

The authors declare that they have no competing interests.

\section{Authors' contributions}

All authors carried out the proof. All authors conceived of the study, and participated in its design and coordination. All authors read and approved the final manuscript.

\section{Author details}

${ }^{1}$ Department of Mathematics, Science and Research Branch, Islamic Azad University, Tehran, Iran. ${ }^{2}$ Department of Mathematics, Amirkabir University of Technology, Tehran, Iran. ${ }^{3}$ Department of Mathematics, Research Institute for Natural Sciences, Hanyang University, Seoul, 133-791, Korea. ${ }^{4}$ Department of Mathematics, Iran University of Science and Technology, Tehran, Iran. ${ }^{5}$ Dipartimento di Matematica e Informatica, Università degli Studi di Palermo, via Archirafi 34, Palermo, 90123, Italy.

\section{Acknowledgements}

The authors would like to thank the referees for giving useful suggestions and comments for the improvement of this paper

Received: 14 November 2013 Accepted: 1 April 2014 Published: 06 May 2014

\section{References}

1. Kramosil, I, Michalek, J: Fuzzy metric and statistical metric spaces. Kybernetika 11, 336-344 (1975)

2. George, A, Veeramani, P: On some results in fuzzy metric spaces. Fuzzy Sets Syst. 64, 395-399 (1994)

3. Deng, Z: Fuzzy pseudometric spaces. J. Math. Anal. Appl. 86, 74-95 (1982)

4. Kaleva, O, Seikkala, S: On fuzzy metric spaces. Fuzzy Sets Syst. 12, 215-229 (1984)

5. Ćirić, L: Some new results for Banach contractions and Edelestein contractive mappings on fuzzy metric spaces. Chaos Solitons Fractals 42, 146-154 (2009)

6. Di Bari, C, Vetro, C: Fixed points, attractors and weak fuzzy contractive mappings in a fuzzy metric space. J. Fuzzy Math. 13, 973-982 (2005)

7. Grabiec, M: Fixed points in fuzzy metric spaces. Fuzzy Sets Syst. 27, 385-389 (1988)

8. Gregori, V, Sapena, A: On fixed point theorems in fuzzy metric spaces. Fuzzy Sets Syst. 125, 245-252 (2002)

9. Miheț, D: A Banach contraction theorem in fuzzy metric spaces. Fuzzy Sets Syst. 144, 431-439 (2004)

10. Miheţ, D: Multivalued generalizations of probabilistic contractions. J. Math. Anal. Appl. 304, 464-472 (2005)

11. Miheţ, D: On the existence and the uniqueness of fixed points of Sehgal contractions. Fuzzy Sets Syst. 156, 135-141 (2005)

12. Saadati, R, Park, JH: On the intuitionistic fuzzy topological spaces. Chaos Solitons Fractals 27(2), 331-344 (2006)

13. Saadati, R, Razani, A, Adibi, H: A common fixed point theorem in L-fuzzy metric spaces. Chaos Solitons Fractals 33(2), 358-363 (2007). doi:10.1016/j.chaos.2006.01.023

14. Sedghi, S, Altun, I, Shobe, N: Coupled fixed point theorems for contractions in fuzzy metric spaces. Nonlinear Anal., Theory Methods Appl. 72(3-4), 1298-1304 (2010)

15. Pathak, HK, Hussain, N: Common fixed points for Banach pairs with applications. Nonlinear Anal. 69, 2788-2802 (2008)

16. Rodríguez-López, J, Romaguera, S: The Hausdorff fuzzy metric on compact sets. Fuzzy Sets Syst. 147, 273-283 (2004)

17. Hadžić, O, Pap, E: Fixed point theorem for multivalued mappings in probabilistic metric spaces and an application in fuzzy metric spaces. Fuzzy Sets Syst. 127, 333-344 (2002)

18. Kiani, F, Amini-Harandi, A: Fixed point and endpoint theorems for set-valued fuzzy contraction maps in fuzzy metric spaces. Fixed Point Theory Appl. 2011, 94 (2011)

19. Miheţ, D: A class of contractions in fuzzy metric spaces. Fuzzy Sets Syst. 161, 1131-1137 (2010)

20. Tirado, P: Contraction mappings in fuzzy quasi-metric spaces and $[0,1]$-fuzzy posets. In: VII Iberoamerican Cont. on Topology and Its Applications, Valencia, Spain, pp. 25-28 (2008)

21. Latif A, Beg, I: Geometric fixed points for single and multivalued mappings. Demonstr. Math. 30(4), 791-800 (1997)

22. Altun, I, Turkoglu, D: Some fixed point theorems on fuzzy metric spaces with implicit relations. Commun. Korean Math. Soc. 23(1), 111-124 (2008)

23. Beg, I, Butt, AR: Fixed point for set-valued mappings satisfying an implicit relation in partially ordered metric spaces. Nonlinear Anal. 71, 3699-3704 (2009)

24. Popa, V: A general coincidence theorem for compatible multivalued mappings satisfying an implicit relation. Demonstr. Math. 33, 159-164 (2000) 
25. Sedghi, S, Rao, KPR, Shobe, N: A general common fixed point theorem for multimaps satisfying an implicit relation on fuzzy metric spaces. Filomat 22(1), 1-11 (2008)

26. Schweizer, B, Sklar, A: Statistical metric spaces. Pac. J. Math. 10, 314-334 (1960)

27. Hadžić, O, Pap, E: Fixed Point Theory in Probabilistic Metric Space. Kluwer Academic, Dordrecht (2001)

28. Hadžić, O, Pap, E, Budinčević, M: Countable extension of triangular norms and their applications to the fixed point theory in probabilistic metric spaces. Kybernetika 38(3), 363-381 (2002)

10.1186/1029-242X-2014-157

Cite this article as: Sadeghi et al.: Set-valued mappings in partially ordered fuzzy metric spaces. Journal of Inequalities and Applications 2014, 2014:157

Submit your manuscript to a SpringerOpen ${ }^{\circ}$ journal and benefit from:

- Convenient online submission

- Rigorous peer review

- Immediate publication on acceptance

Open access: articles freely available online

- High visibility within the field

- Retaining the copyright to your article

Submit your next manuscript at $>$ springeropen.com 